\title{
Surface ionizing dose for space applications estimated with low energy spectra going down to hundreds of $\mathrm{eV}$
}

\author{
C. Inguimbert, P. Caron, Q.Gibaru, A. Sicard, N. Balcon, and R. Ecoffet
}

\begin{abstract}
The contribution of low energy particles down to $\sim 200 \mathrm{eV}$ to the dose deposited on the very near surface of materials subject to the space environment is investigated by means of GEANT4 Monte Carlo simulations. The contribution to the dose, of the low energy parts of GREEN spectra ([200 eV-40 $\mathrm{keV}$ ] for electrons, [200 eV-100 keV] for protons) are compared with calculations performed with AE8/AP8. Both GEO and LEO SPOT like orbits are studied. The dose depth profiles are estimated for silicon material. The impact on the dose calculation of different transport models is also investigated. Below $1 \mathrm{keV}$, the relevance of continuous processes is analysed for electrons by comparison with a discrete model (GEANT4/MICROELEC). This analysis is also performed for protons below $10 \mathrm{keV}$.
\end{abstract}

Index Terms - Space Environment, Total ionizing dose, Dose depth profile, electron, proton.

\section{INTRODUCTION}

$\mathrm{T}$ The radiative constraint is commonly estimated for space projects via the calculation of the ionizing dose depth profiles. It is a combination of energetic spectra of different particles (electrons, protons) considering their respective ionization capabilities. The calculation of the ionizing dose is very well-known and managed in the field of space applications. Parameter tables like LET (Linear Energy Transfer $=\mathrm{dE} / \mathrm{dx}$ ) allow quick first order calculations, approximating the dose as the product of the incident fluence by the LET of the respective particles. In the space domain, the most commonly used LET tables are the SRIM [1], [2] or the NIST [3], [4] tables. More or less sophisticated calculation models exist in the literature to perform ionizing dose estimations. For example, Shieldose [5], [6] is a very powerful calculation tool, which allows quick and precise calculations for simple geometries, thanks to the use of tabulated parameters. For example, the OMERE software [7] uses this database. Even simpler analytical methods exist, as for example the work of Bortfeld [8], for protons in the medical field. Similar attempts can be found in the space domain. However, more accurate calculations require more sophisticated transport codes, based on the Monte Carlo method. SRIM [1] is a very commonly used toolkit for protons and heavy ions. The CREME-MC [9], [10] software, dedicated to the estimation of the Single Event Effects rates (SEE) in microelectronics, includes an ionizing dose calculation module for protons. For space projects, the use of Monte Carlo (MC) codes such as NOVICE [11], [12] or FASTRAD [13] is widespread. MULASSIS code [14] of

Ma,ucript received Sept. 24, 2020

C. Inguimbert, P. Caron, Q.Gibaru, and A. Sicard, are with ONERA 2.av. E. Belin 31055 Toulouse, France (e-mail: christophe.inguimbert@onera.fr tel: +33- 562252734).

N. Balcon, R. Ecoffet and Q.Gibaru are with CNES 18 av. E. Belin, 31055 Toulouse, France.

Q.Gibaru is with CEA, DAM, DIF - 91297 Arpajon, France.
SPENVIS interface [15] can also be used. This latter is developed with GEANT4 [16], [17], a generic radiation transport tool, which can also more specifically be used to estimate the dose. In this kind of toolkit, the transport of particles is carried out step by step. The energy losses, and therefore the energies deposited into the target material, are evaluated along the trajectory of each individual particle. The dose is estimated as an average value deduced from the transport of a significant number (several thousands) of incident particles. These MC methods, unlike tabulated or analytical calculations, allow the dose to be calculated in complex geometries, but are obviously much more time consuming.

Estimating the dose at the surface of the satellites is another concern for space projects. All external surfaces of satellites are exposed to high radiation levels, which are actually poorly estimated. These high levels of ionizing radiations strongly degrade the thermo-optical properties of surface coatings, such as white paints, Kapton, Optical Solar Reflector (OSR), and MLI (Multi Layer Insulator). Degradations can be observed typically from 0.1 MGy, while deposited doses at the surface, depending on the orbit, are more in the order of $1 \mathrm{MGy} / \mathrm{year}$. This is true for both thermo-optical properties of coatings and conduction properties of insulator materials [18], [19]. For example, yellowing induces additional absorption levels that increase the solar absorptivity and thus the equilibrium temperature of the illuminated systems. This increases the amplitude of thermal cycling which can have negative knockon effect on the equipment. For instance, antennae which are generally covered with white paints, are very sensitive to potential deformations (defocus). The information provided by optical systems can thus be altered by the production of color centers. The cover glasses of solar panels are also critical items particularly sensitive to radiation. The degradation of anti-reflection treatments, of a few tens of nanometers thick, produces additional absorption levels that contribute to reduce the supplied power. Another example is the aging of the adhesives. The modification of their electrical properties increases the risk of electrical discharges (potentially destructive). Most of the thermo-optical properties lie in the first hundreds of nanometers of the materials (typically of the order of the light wavelength). It is thus of critical importance to accurately evaluate the radiation level received at the surface of these materials $(<\mu \mathrm{m})$, whereas the dose is commonly estimated at best at the first micron. For instance OMERE provides dose computations at a minimum depth of $10 \mu \mathrm{m}$ while MULASSIS pushes the calculation down to 0.5 $\mu \mathrm{m}$. These limits are induced by the self-limitation of the continuous processes used in those toolkits, which are not suited for low energies $(<\sim 1 \mathrm{keV})$ and small scales $(<\sim \mu \mathrm{m})$. The estimation of surface doses faces two main issues. First of all, the incident energetic spectra for both 
protons and electrons are not known at low energy. The lower limits of respectively AP8 and AE8 [20], [21] are $100 \mathrm{keV}$ and $40 \mathrm{keV}$. The exponential decay of the environmental spectra means that the dose on the surface of the materials exposed to the space environment is largely dominated by low energy particles $(<\mathrm{keV})$. In addition, the materials optical properties are determined by the first hundreds of nanometers, while the dose is commonly estimated at best in the first micron. A relevant estimation of the surface dose at these typical depths requires to account for the low energy particles of the environment, and to use radiation transport codes valid down to those energies (typically a few tens of eV). Which brings us to the second point: the conventional radiation transport codes listed above, based on continuous approximation, reach their limit of validity at such energy levels. Luckily, recent improvements in this field allow investigating the accuracy of the dose calculation close to the surface of the materials. New environment models valid down to a few hundreds of $\mathrm{eV}$ have been developed and are available for the space community. NASA provides the AE9/AP9 [22], [23] model. ONERA with
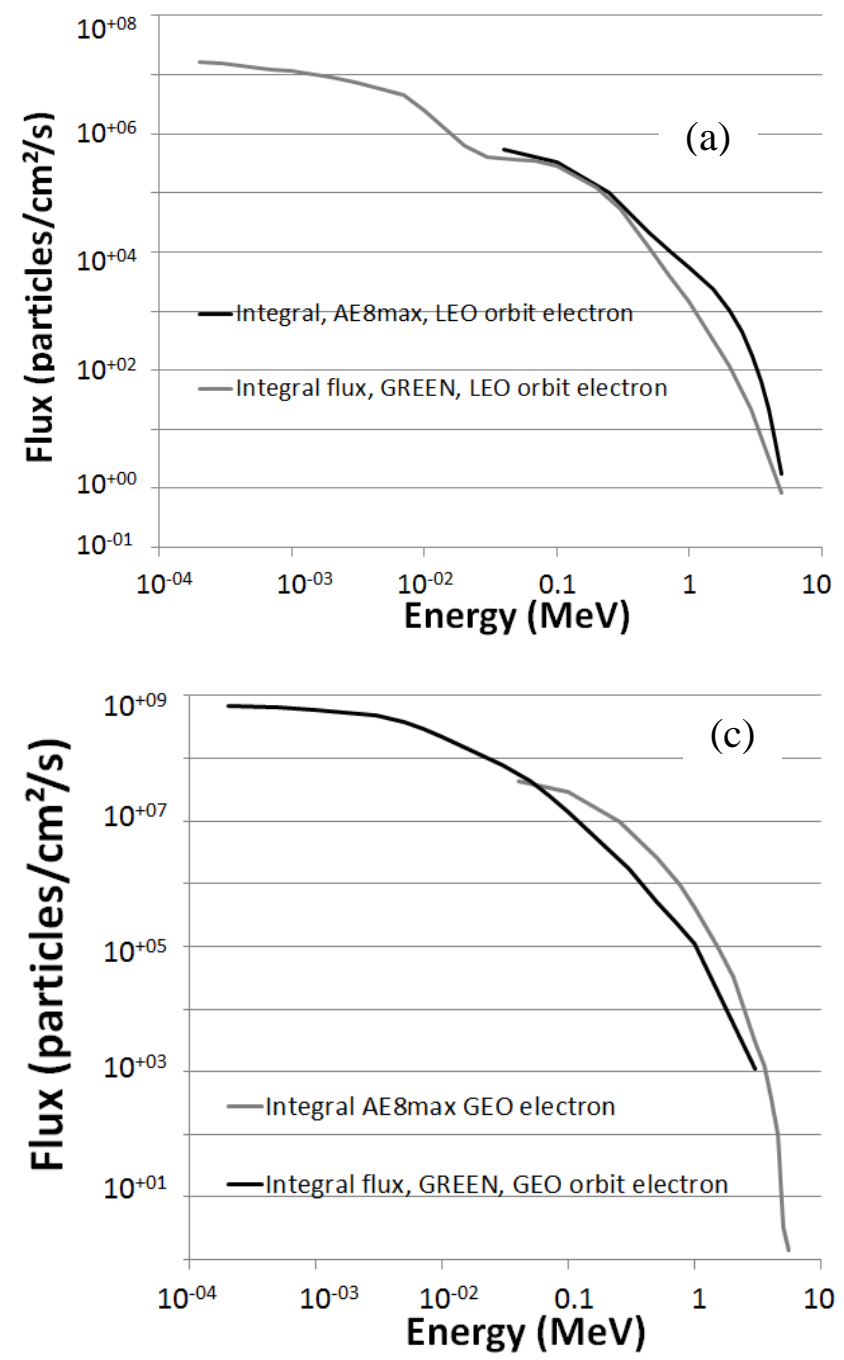

the support of the French Space Agency (CNES), has also developed the GREEN [24] model, with cutoff energies of 200 $\mathrm{eV}$. In addition, radiation transport models valid down to a few $\mathrm{eV}$ for electrons and a few tens of $\mathrm{keV}$ for protons have been recently developed and made available to the space community [25]-[33].

The objective of the present study is to assess the impact of low energies ( $<40 \mathrm{keV}$ for electrons, $\sim 100 \mathrm{keV} / \mathrm{uma}$ for ions) on the evaluation of the ionizing dose close to the materials surface $(\sim 10 \mathrm{~nm})$. The dose depth profiles are calculated for two standard missions (a spot type Low Earth Orbit - LEO and a Geostationary Earth Orbit - GEO), whose environments have been estimated thanks to AE8/AP8 and GREEN models. The importance of the low energy part of the spectrum on the dose depth profile and the need to use dedicated transport codes valid at low energy ( $100 \mathrm{eV}$ for electrons, $\sim 10 \mathrm{keV}$ for protons) are both addressed.

\section{LEO AND GEO ENVIRONMENT SPECTRA}
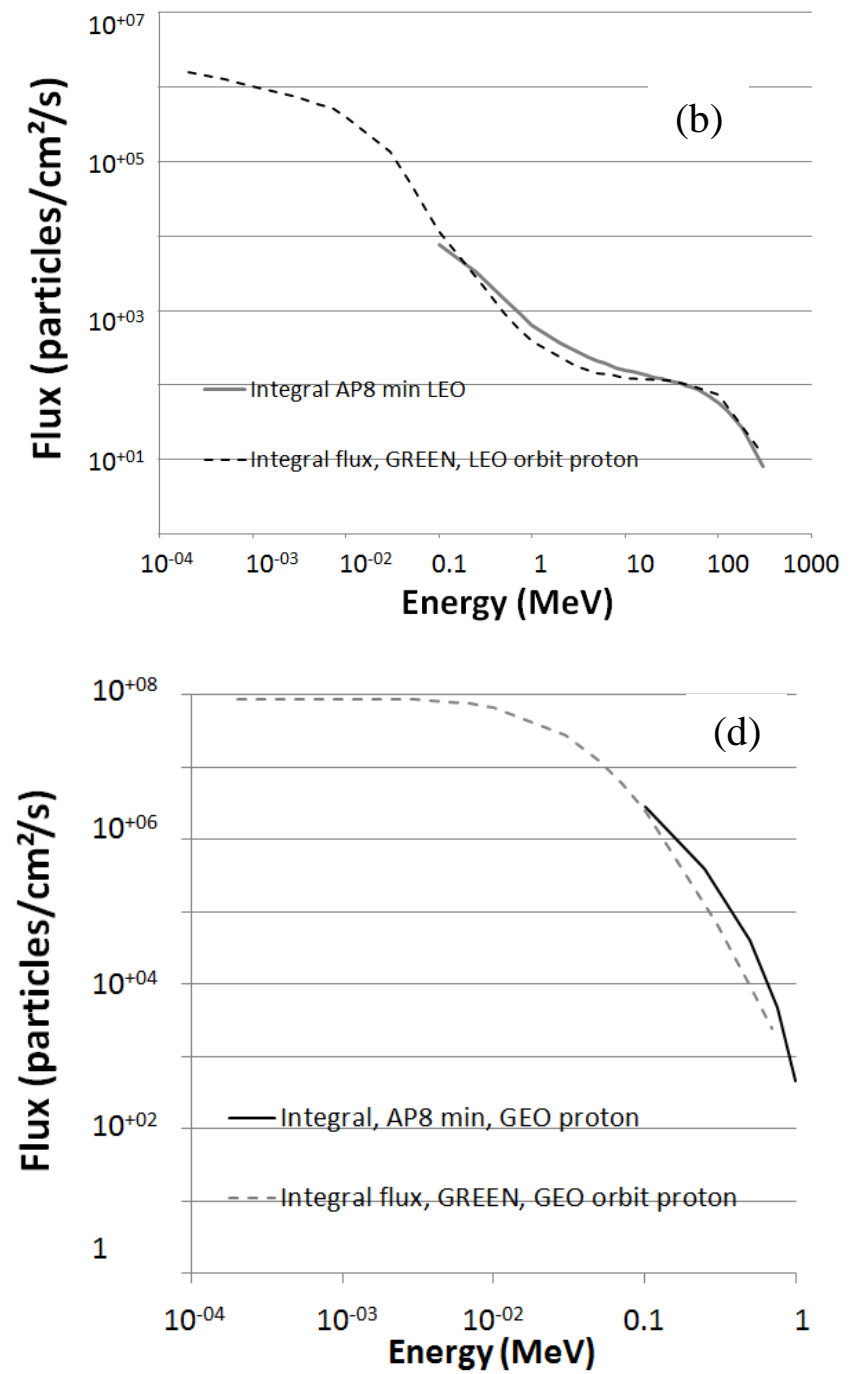

Fig. 1. GREEN comparison with AE8 and AP8 for LEO spot type orbit ((a) for electrons, (b) for protons), and GEO orbit ((c)for electrons and (d) for protons). Below the cutoff energy of AE8 (40 keV) and AP8 $(100 \mathrm{keV})$ the fluxes are multiplied by at least a factor of 20. Above these cutoffs GREEN, AE8 and AP8 provide comparable fluxes, even if some important discrepancies can be observed at certain portions of the spectra. As can be seen in section V, those differences impact dose depth profiles at large depth (depth $>100 \mu \mathrm{m})$. 
Different environmental models describing the radiation belts of the earth exist. The reference models in all international standards are still the NASA AE8 and AP8 [21] models, respectively for electrons and protons, even if some updates (AE9, AP9) [23] and new models have been proposed since (GREEN) [24]. The low energy cutoffs of both models are respectively $40 \mathrm{keV}$ for electrons (AE8) and $100 \mathrm{keV}$ for protons (AP8). AE9 and AP9, like AE8 and AP8, are intended for the design of space systems. This update aims at extending the low energy range of AE8 and AP8 and provides statistical confidence levels. These capabilities are also offered by the ONERA GREEN model [24]. This latter model has been used for this study.

GREEN (Global Radiation Earth ENvironment) is a new global model providing radiation belt fluxes between $\mathrm{L}^{*}=1$ and $\mathrm{L}^{*}=8\left(\mathrm{~L}^{*}=\right.$ radial distance in Earth radii of a field line expressed in the magnetic equator), along the magnetic field lines, for any energy between $200 \mathrm{eV}$ and $10 \mathrm{MeV}$ for electrons and between $200 \mathrm{eV}$ and $800 \mathrm{MeV}$ for protons [24].

The GREEN spectra are compared to those of AE8 and AP8 on two different orbits: the geostationary orbit and the low SPOT orbit $\left(98^{\circ}\right.$ inclination, $800 \mathrm{~km}$ altitude). The environmental conditions chosen for these calculations are:

- AE8: JENSEN_CAIN (1960) magnetic field in solar max.,

- AP8: JENSEN_CAIN (1960) magnetic field in solar min.,

- GREEN: IGRF magnetic field + Olson Pfitzer Quiet conditions.

The integrated fluxes calculated with GREEN for the LEO and GEO orbits are presented and compared to both AE8 and AP8 in Fig. 1. The GREEN's low energy cutoff is $200 \mathrm{eV}$ for both electrons and protons, to be compared respectively to 40 $\mathrm{keV}$ (AE8) and $100 \mathrm{keV}$ (AP8). The addition of the low energy part of the spectra enhances the total fluxes by at least a factor of 20 . More precisely, the fluence is multiplied by a factor of 40 for electrons in LEO orbits $\left(1.6 \times 10^{+7} \mathrm{e}-/ \mathrm{cm}^{2}\right.$ vs. $\left.3.7 \times 10^{+5} \mathrm{e}-/ \mathrm{cm}^{2}\right)$ and by a factor of 200 for protons on the same orbit $\left(1.610^{+6} \mathrm{p} / \mathrm{cm}^{2}\right.$ vs. $\left.7.6 \times 10^{+3} \mathrm{p} / \mathrm{cm}^{2}\right)$. For the GEO orbit, the fluence is multiplied by 20 for electrons $\left(6.9 \times 10^{+8}\right.$ $\mathrm{e}-/ \mathrm{cm}^{2}$ versus $\left.4.310^{+7} \mathrm{e}-/ \mathrm{cm}^{2}\right)$ and by 35 for protons $(8.7 \mathrm{x}$ $10^{+7} \mathrm{p} / \mathrm{cm}^{2}$ versus $2.5 \times 10^{+6} \mathrm{p} / \mathrm{cm}^{2}$ ). One can understand easily that the dose is enhanced on these orbits and specially at the surface of the irradiated materials, where the low energy particles are absorbed by the medium. This is shown in section IV, after a short presentation of the used transport model in section III.

\section{RADIATION TRANSPORT MODEL}

\section{A. Overview}

For dose calculations of space applications, due to CPU time constraint and the complex large 3D geometries considered, the most commonly used transport codes are based on the multiple scattering approximation. This approximation can estimate the particles slow-down along an elementary path by averaging interaction results over each jump between calculated collisions.

This method is valid only if the particle has undergone a sufficient number of "electronic" interactions to ensure a proper calculation of the dissipated energy from the linear energy loss $(\mathrm{dE} / \mathrm{dx})$ of the incident particle, and therefore if the length traveled, over which the energy loss is estimated, is sufficiently large. This approximation is applicable at high energies, since the average energy transfers of a few hundred $\mathrm{eV}$ are low compared to the total energy of the incident particle. The energy loss can in this case be evaluated with a sufficient accuracy over fairly large distances and for a sufficient number of interactions.

At lower energy, typically below $\sim 1 \mathrm{keV}$, the incident energies are of the same order of magnitude as the discrete atomic energetic levels. A single energy transfer between an incident particle and an electron of the medium becomes significant as compared to the incident energy. It becomes difficult within the multiple scattering approximation to find a tradeoff between the energy loss, the step length and the angular deflection. It is then necessary to simulate all the elementary interactions between the impinging particles and the electrons of the atomic shells. The more energetic the particle is, the slower the calculation is because the number of interactions to be simulated increases in proportion to the incident energy. The time duration could be a real issue for space applications where the transport of energetic particles, that can reach several hundred $\mathrm{MeV}$ (protons), must be performed in big geometries. In that scope, the use of multiple scattering processes is to be preferred to slower single scattering processes.

In addition, at low energy $(<\mathrm{keV})$, the transport of particles is not straightforward. Indeed, below a few hundreds of eV, the interaction processes are extremely dependent on the electronic structure of the target materials. The processes are driven at low energy by interband transitions which magnitudes depend closely on the energy bandgap of the materials. Metals, semiconductors and insulators behave quite differently. There is therefore a limit to the validity of the current transport models when used at low energy. Actually, this limit is of the order of $\sim 1 \mathrm{keV}$ for electrons, and of a few hundreds of $\mathrm{keV}$ per atomic unit for ions. Some models have been specifically developed to address the low energy domain (typically below $\sim \mathrm{keV}$ for electrons). For instance, PENELOPE [25] is valid down to $250 \mathrm{eV}$. Some other models based on the dielectric function theory have also been developed [26]-[33], which allow extending the validity domain down to a few eV. These developments are however adapted on a case-by-case basis for each material and have only been applied in the literature to a very limited number of materials [26]-[33]. Up to now, it was not a real issue for space applications, as the environment models such as AE8 and AP8 [21] presented cutoff energies far above the low energy limit of the transport methods. This is no longer the case with GREEN and AE9/AP9 environment models which provide energetic spectra down to a few hundred $\mathrm{eV}$. Improving the ionizing dose calculations, at small scale close to the surface, requires the use of relevant transport models valid at least down to a few hundred $\mathrm{eV}$.

\section{B. Different low energy modules in GEANT4}

GEANT4 offers a wide selection of physical models. In most cases, for a given physical process, the user can choose at least between two distinct models. For electromagnetic physics (EM), there are standard EM processes, above $1 \mathrm{keV}$, 
and low energy EM processes, valid down to $\sim 250 \mathrm{eV}$ for electrons and gamma rays. The electromagnetic processes contribute mainly to the slowing down of incident particles while the other processes ("hadronic") deal with the nuclear potential interactions. The latter are out of the scope of this study. We will therefore focus on the different models describing the electromagnetic interactions. In GEANT4, we can distinguish for electrons, four different electromagnetic processes applicable in different energy ranges:

- G4eIonization (STANDARD)

- G4LivermoreIonisationModel (LIVERMORE)

- G4PenelopeIonisationModel (PENELOPE)

- G4MicroElec (MICROELEC)

G4eIonization (STANDARD) physics is a continuous process valid above $1 \mathrm{keV}$. Similarly, according to the GEANT4 documentation [17], the Livermore continuous ionization model can be used down to few tens of eV. The PENELOPE (PENetration and Energy LOss of Positrons and Electrons) model is also a continuous ionization process which is valid down to $\sim 100 \mathrm{eV}$ [25]. These continuous inelastic processes must be combined with some elastic interaction models to account for the scattering of particles on nuclei. Similarly, these later processes can be discrete (Single Scattering : SS) or "averaged" (Multiple SCattering : Msc). GEANT4 provides some scenarios combining inelastic processes with single (SS) or multiple elastic scattering (Msc) processes. For instance, in order to increase the accuracy of the standard processes, the G4EmStandardPhysicsSS scenario combines the standard (Std) ionization process with the single elastic process (SS). For its part, The G4EmStandardPhysics_option4 combines different models according to the nature and the energy of the incident particles. For low energy electrons this scenario combines the PENELOPE continuous ionization process with the multiple scattering processes. In order to improve the accuracy, the multiple scattering processes are replaced by the single scattering process for large deviation angles (Msc/SS in Table I). The G4EmLivermorePhysics differs from the G4EmStandardPhysics_option4 scenario by replacing the PENELOPE ionization process with the LIVERMORE ionization process. A summary of the different scenarios is presented in Table I.

Table I

DIFFERENT GEANT4 PHYSIC LISTS
\begin{tabular}{|c|c|c|c|c|}
\hline Physics List & \multicolumn{2}{|c|}{ ionization } & \multicolumn{2}{c|}{ Elastic scattering } \\
\hline & Electron & Proton & Electron & Proton \\
\hline G4EMStandardPhysics & Std & Std & Msc/SS & Msc/SS \\
\hline G4EMLivermorePhysics & Liv $(<100 \mathrm{keV})$ & Std & Msc/SS & Msc/SS \\
\hline G4EmStandardPhysics_opt4 & Pen $(<1 \mathrm{MeV})$ & Std & Msc/SS & Msc/SS \\
\hline Penelope+ELSEPA & Pen $(<1 \mathrm{MeV})$ & - & SS & $-*$ \\
\hline G4EmStandardPhysicsSS & Std & Std & SS & SS \\
\hline
\end{tabular}

The best accuracy can be reached by using discrete processes for both ionization and elastic scattering. The MICROELEC [26]-[31] module of GEANT4, provides such algorithms. It allows the transport of electrons down to a few $\mathrm{eV}$. Some improvements have also been brought to allow the transport of protons down to $\sim 10 \mathrm{keV} \mathrm{[32].}$
The accuracy of some of these models for the dose calculation at the very near surface of irradiated materials is studied. Results are presented in sections IV and V. The comparison is also made with some other transport codes presented in the following section.

\section{Other codes}

For comparison, in some cases, the ionizing doses have also been estimated thanks to SRIM [1], MULASSIS [14] and OMERE [7], three different toolkits commonly used within the space community. MULASSIS is a GEANT4 based application whose physics have been optimized for the dose depth profile calculations. The calculation is valid for protons down to $10 \mathrm{keV}$. It is based on the multiple scattering (G4UrbanMscModel) approximation for elastic interactions. The standard ionization (G4eIonization, G4hIonization) processes are used for electrons and protons respectively. OMERE is a general framework including models dedicated to the study of radiation effects on space systems [7]. A transport module allowing dose depth profile calculations is provided. It is based on the SHIELDOSE database [5], [6]. Its advantage is speed; its drawback is the limitation at low energy. SRIM [1] has also been used as a reference for low energy incident proton beams.

\section{COMPARISON OF THE DIFFERENT TRANSPORT MODELS}

The dose is calculated for low energies and small dimensions with different models in order to investigate, the relevance of continuous transport processes. Continuous based models are compared to calculations that use discrete transport methods (MICROELEC and OSMOSEE [30], [31]). The anlysis is focused on two GEANT4 scenarios:

- G4EmStandardPhysics_option4,

- G4LivermoreIonisationModel).

Dose depth profiles have been calculated in silicon target (semi infinite slab) for both protons and electrons. Calculations have been performed down to respectively $2 \mathrm{keV}$ and $200 \mathrm{eV}$ for normal incident protons and electrons. It should be noted that the calculation neglects some physical aspects. Rigorously, an accurate calculation of the dose near the surface must take into account the surface state including the roughness, the presence of dangling bonds, defects, and the distortion of the band structure, which at very low energy could play an important role in the energy transfer process. All these mechanisms have been neglected in our dose depth profile calculations.

\section{A. Monokinetic normal incident electrons}

The GEANT physical scenario studied by default is the G4EmStandardPhysics_option4 module that is a good tradeoff between speed and accuracy at low energy (cf. §III.B, Table I). The calculations have been performed in silicon targets for normal incident electron beams having energies from $200 \mathrm{eV}$ up to $57 \mathrm{keV}$. The dose depth profile is estimated from the maximum penetration depth down to $1 \mathrm{~nm}$ close to the surface. In Fig. 2, the calculations have been validated at high energy $(57 \mathrm{keV})$, by comparison with experimental data of ref. [34] measured in aluminium material. For comparisons with the calculation performed in silicon material, the dose depth profile measured in $\mathrm{Al}\left(\operatorname{Dose}_{\mathrm{Al}}\left(\mathrm{x}_{\mathrm{Al}}\right)\right)$ has been rescaled 
according to the density of the silicon material $\left(\rho_{\mathrm{Si}}\right)$ following the expression $\operatorname{Dose}_{\mathrm{Al}}\left(\mathrm{x}_{\mathrm{Si}}\right)=\operatorname{Dose} \mathrm{Al}\left(\mathrm{x}_{\mathrm{Al}} \times \rho_{\mathrm{Al}} / \rho_{\mathrm{Si}}\right)$, with $\rho_{\mathrm{Al}}=$ $2.7 \mathrm{~g} / \mathrm{cm}^{3}$ and $/ \rho_{\mathrm{Si}}=2.33 \mathrm{~g} / \mathrm{cm}^{3}$.

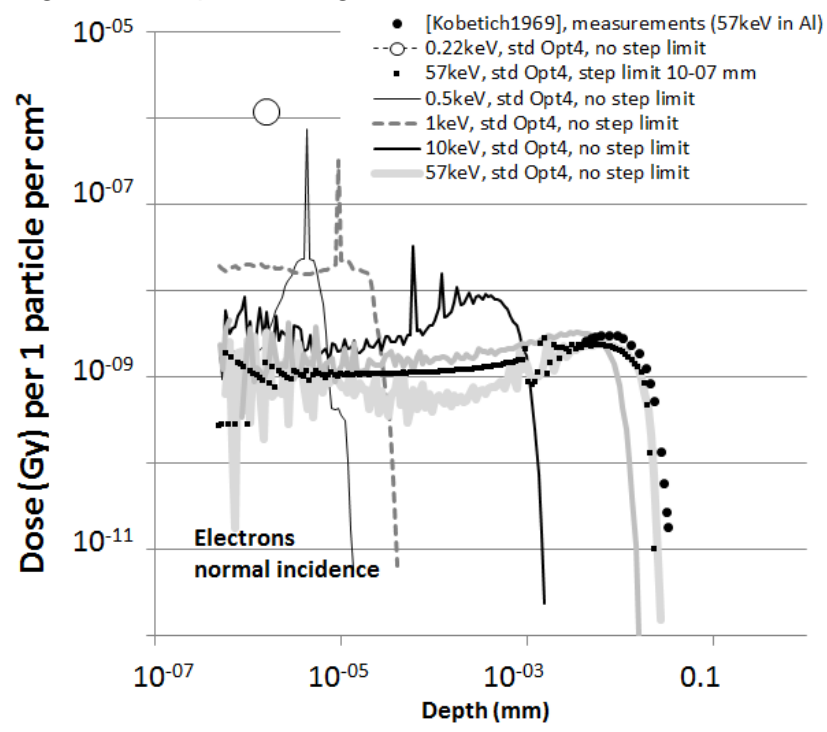

Fig. 2. Electron in silicon dose depth curve. Calculation are performed with G4EmStandardPhysics_option4 model of GEANT4 with normal incident electrons having energies in the range [220 eV, $57 \mathrm{keV}]$.

In the case of $57 \mathrm{keV}$ electrons two calculations have been performed, one with no step limitation and the second one with a step limitation in the first micron of the volume $\left(10^{-07}\right.$ $\mathrm{mm})$. The agreement with experimental data is good, even if the calculation is noisy close to the surface, where the sample bins are too small to get a statistical convergence. The step limitation could be a good way to increase the accuracy of Monte Carlo radiation transport calculations. Here a semiinfinite silicon slab has been topped with a thin silicon overlayer of $1 \mu \mathrm{m}$ thickness. In this latter region, the step length can be tuned to improve the accuracy of the dose calculation near the surface.

The case, for which the step length is limited to 1 Angström $\left(10^{-7} \mathrm{~mm}\right)$ in the first micron is far less noisy. However, one should note the presence of an artifact at $1 \mu \mathrm{m}$ depth, at the interface between the two volumes, where there is on one side a step limitation and on the other side no step limitation. This shows clearly that particular care must be taken to the step length parameter.

In Fig. 2, the rest of the simulations have been performed without any tuning of the step limitation. The $40 \mathrm{keV}$ case seems to be reasonable with no step limitation, while at lower energy some unexpected dose peaks appear. The shape of the dose depth profile becomes worse as the energy deceases. The $500 \mathrm{eV}$ case has a very atypical shape, the $220 \mathrm{eV}$ (low energy limit of the PENELOPE model) provides a single point (circle), clearly showing the real limitation of PENELOPE.

The connection between the peaks shown in Fig. 2 and the step length is demonstrated in Fig. 3. For $1 \mathrm{keV}$ incident electrons, the peaks are removed using a very small step length of 1 Angström. But this seems to degrade the dose depth profile calculation, which more and more deviates from the reference calculation of MICROELEC. The range is half, and the dose level is twice the values calculated with MICROELEC (Fig. 3). In that case increasing the accuracy of the transport modelling by limiting the step, degrades the result. Low energy transport of electrons is also driven by the elastic scattering process. In the case of the StdPhys-Opt4 scenario of Fig. 3, the degradation of the results induced by limiting the step size is due to the elastic process involved in this scenario, which seems not to be very good below some $\mathrm{keV}$. Combining the ionizing process of StdPhys-Opt4 scenario (Penelope) with the elastic process of microelec (ELSEPA) makes the two calculations converge (Fig. 4).

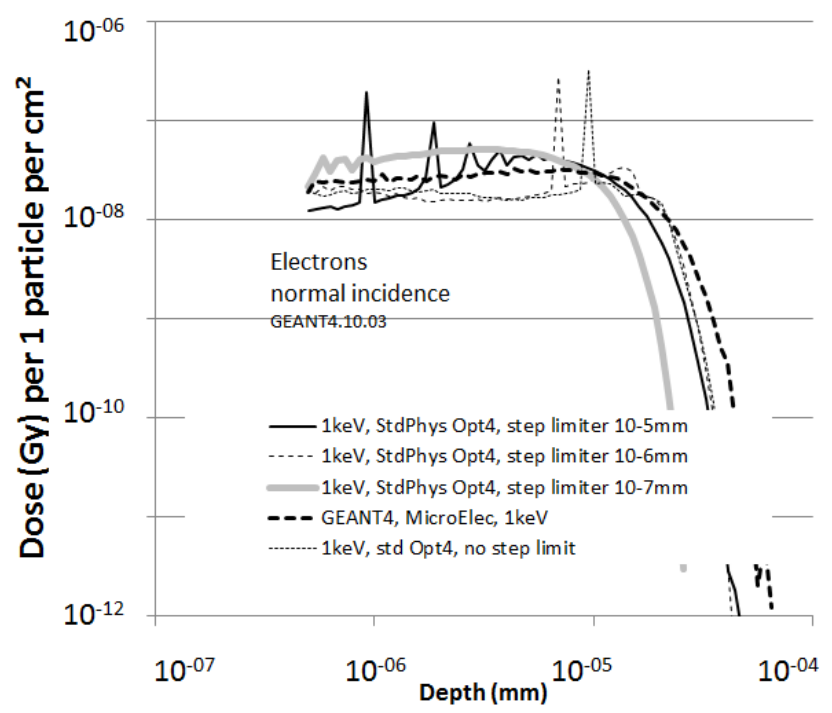

Fig. 3. Normal incident $1 \mathrm{keV}$ electron in silicon dose depth curve. Calculations are performed with G4EmStandardPhysics_option4 model using different step limit from 1 Angström $\left(10^{-7} \mathrm{~mm}\right)$ up to $10 \mathrm{~nm}\left(10^{-5} \mathrm{~mm}\right)$. Comparison is performed with MICROELEC discrete process of GEANT4.

For $1 \mathrm{keV}$ incident electrons, the comparisons of the dose depth curves estimated with different models is shown in Fig. 4. The two discrete models MICROELEC [26]-[29] and OSMOSEE [30] are very close to each other. In both cases the elastic processes are simulated according to the ELSEPA cross sections [35], while the standard multiple scattering process of GEANT4 is used in G4EmStandardPhysics_option4 and the Livermore scenario. The use of ELSEPA scattering with PENELOPE ionizing process (Fig. 4) provides results in very good agreement with the discrete models.

ELSEPA [35] describes the elastic scattering of electrons by a local central interaction potential. The code is a solver based on a Dirac partial-wave formalism. The nucleus is described by a Fermi distribution and the electronic density is based on the Dirac-Fock formalism. The accuracy of the calculation is thus demonstrated being closely dependent on the choice of both ionizing and elastic processes.

To conclude, this parametric study underlines the fact that the discrete processes are often better suited than the continuous processes for low energy electron dose calculations. It clearly shows the importance of a proper simulation of the elastic processes, which directly impact the range of the electrons and thus the deposited dose. The simulations also show that the accuracy of the calculation depends closely on the used step length. But a good accuracy can be reached with continuous ionizing processes, if precise electronic stopping powers are used, in combination with a relevant single elastic process. For instance, the PENELOPE 
ionization process provides good results down to very low energy, when it is combined with the ELSEPA elastic model. The agreement between continuous PENELOPE+ELSEPA and MICROELEC+ELSEPA models is shown to be good for $1 \mathrm{keV}$ electrons (Fig. 4). This agreement remains good down to $300 \mathrm{eV}$ (Fig. 5).

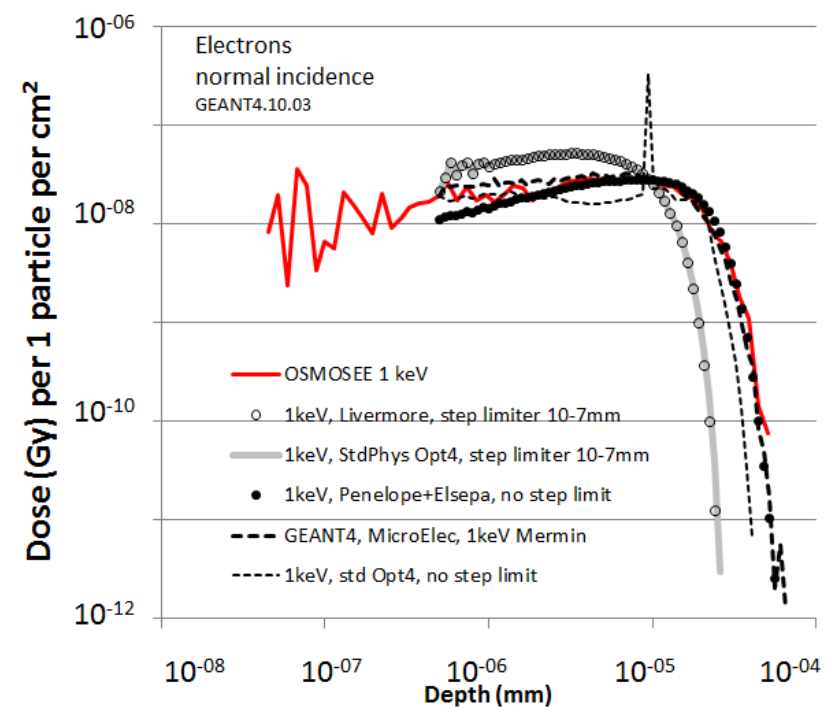

Fig. 4. Normal incident $1 \mathrm{keV}$ electrons in silicon dose depth curves. G4EmStandardPhysics_option4, Livermore, PENELOPE+ELSEPA, and MICORELEC models are compared.

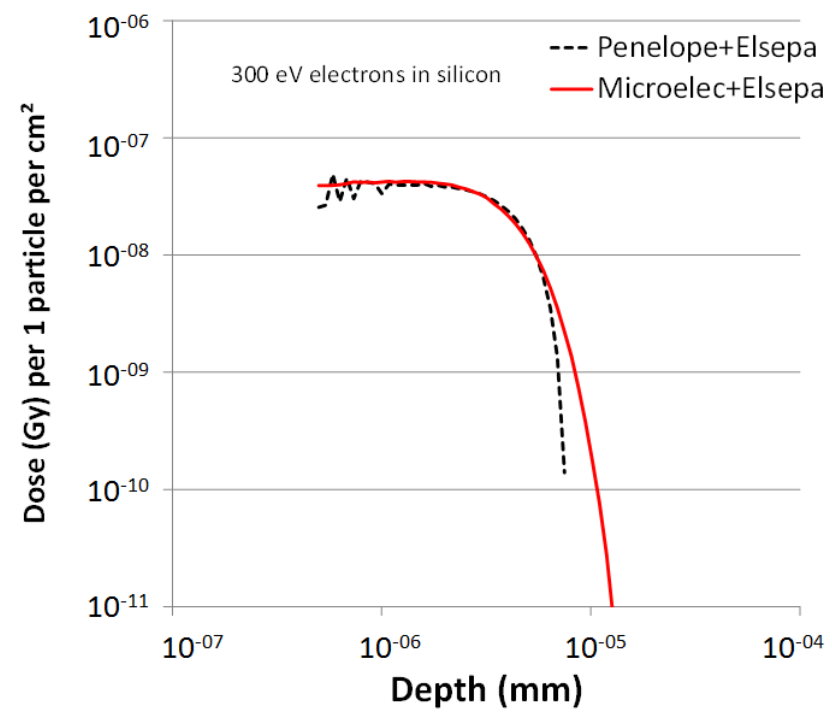

Fig. 5. Normal incident $300 \mathrm{eV}$ electrons in silicon dose depth curves. PENELOPE+ELSEPA and MICORELEC+ELSEPA models are compared. The PENELOPE continuous process provides results close to MICROELEC discrete process down to several hundreds of $\mathrm{eV}$.

\section{B. Monokinetic normal incident protons}

The dependence of the accuracy of the dose calculation on the accuracy of the electronic stopping power, is even more obvious for protons. In this part, dose depth profiles have been calculated in silicon for normal incident $100 \mathrm{keV}$ and $2 \mathrm{keV}$ protons (Figs. 6 and 7). Like previously for electrons, different models and the use of different step lengths are compared.

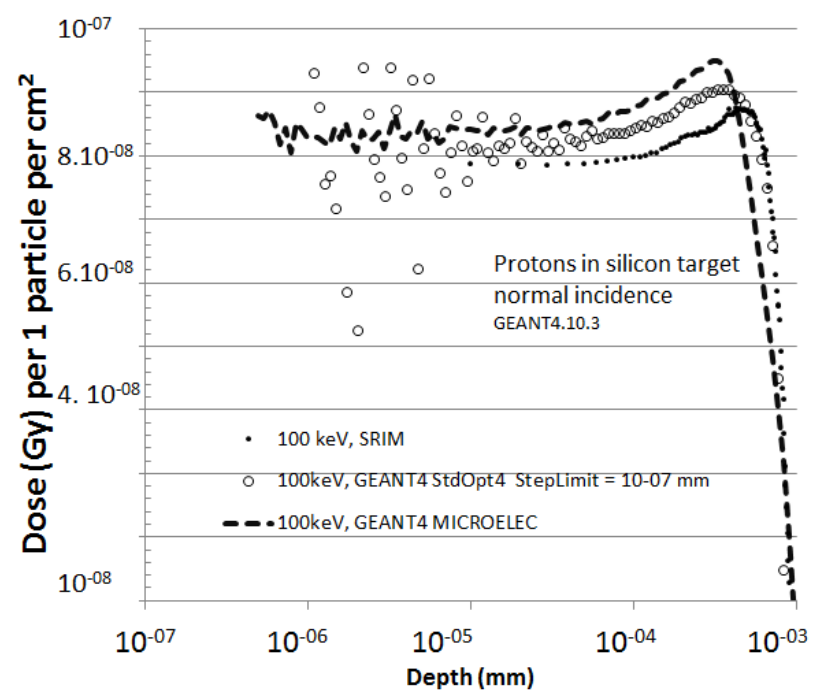

Fig. 6. Normal incident $100 \mathrm{keV}$ protons in silicon dose depth curve. G4EmStandardPhysics_option4, MICORELEC and SRIM models are compared. The agreement is quite good.

G4EmStandardPhysics_option4 MICROELEC and SRIM are compared on Figs. 5 and 7. At $100 \mathrm{keV}$ all models are based on very close stopping powers. One can observe on Fig. 5 less than $10 \%$ difference on the average dose level between MICROELEC and SRIM, and about $2.5 \%$ difference between G4EmStandardPhysics_option4 and SRIM. SRIM is chosen as a reference as it is based on an extensive set of experimental measurements. However, the continuous standard process of GEANT4 is more noisy below $\sim 100 \mathrm{~nm}$ depth, even by using a very short step length of $0.1 \mathrm{~nm}\left(10^{-7} \mathrm{~mm}\right)$, which is optimized to get the best possible result.

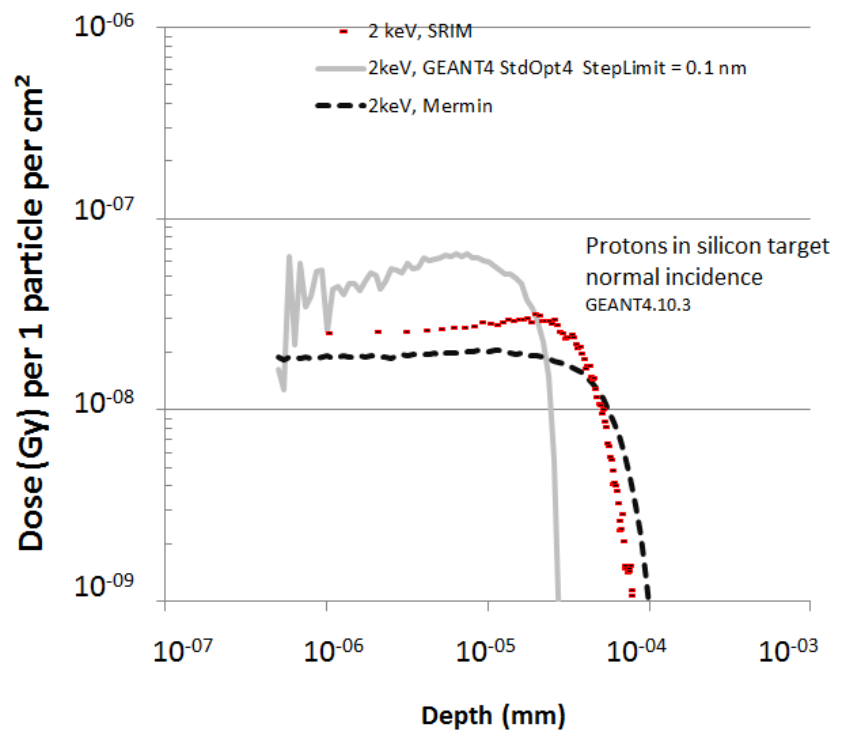

Fig. 7. Normal incident $2 \mathrm{keV}$ protons in silicon dose depth curve. The G4EmStandardPhysics_option4 overestimates the dose by a factor of 2 compared to SRIM. There is only $25 \%$ difference between the SRIM and MICROELEC models.

But at lower energy ( $2 \mathrm{keV}$, Fig. 6) some more significant deviations appear between the different models. Compared to SRIM, the G4EmStandardPhysics_option4 overestimates the dose by a factor of 2 . A difference of only $25 \%$ can be 
observed between SRIM and MICROELEC model that have closer LET vs. energy curves. Once again the discrete processes such as MICROELEC are more relevant at low energy.

\section{Case of isotropic proton energetic spectrum}

As shown in the previous section, at low energy below some $\mathrm{keV}$, significant differences can be observed from a dose calculation to another. A factor of two between SRIM and G4EmStandardPhysics_opt4 (1 Å step limit) has been shown. In that section, we would like to go further by estimating the uncertainties for an isotropic incident energetic spectrum. Same orders of magnitude have been observed for both protons and electrons that depict comparable uncertainties. Only the case of protons is shown here.

For incident protons, the ionizing dose profiles have been calculated for the LEO environment $\left(800 \mathrm{~km}, 98^{\circ}\right)$. The G4EmStandardPhysics_option4 continuous model, with three step limit conditions (no step limit, 1 Angström $\left(10^{-7} \mathrm{~mm}\right), 10$ $\mathrm{nm}\left(10^{-5} \mathrm{~mm}\right)$ ) has been compared to the MICROELEC discrete model. The calculations are relatively slow. So, to limit computation times, the incident proton spectrum has been truncated above $1 \mathrm{MeV}$. The incident proton LEO spectrum ranges from $200 \mathrm{eV}$ to $1 \mathrm{MeV}$. The proton flux greater than $1 \mathrm{MeV}$ only represents $0.02 \%$ of the total spectrum, therefore neglecting it does not affect the dose calculation at the surface of the material.

The importance of limiting the step length of particles is confirmed in Fig. 8. Even if the right order of magnitude is found when using no step limitation (grey curve in Fig. 8), one can notice a worse agreement with the MICROELEC discrete calculation. Forcing a step limit to $10 \mathrm{~nm}\left(10^{-5} \mathrm{~mm}\right)$ improves significantly the result. The calculation can even more be improved by reducing this step limit to $1 \AA\left(10^{-7} \mathrm{~mm}\right)$. Above $10 \mathrm{~nm}$ depth, the MICROELEC and G4EmStandardPhysics_option4 $\left(1 \AA\left(10^{-7} \mathrm{~mm}\right)\right)$ calculations are superimposed. Below there is again a factor of 2 difference between the calculations. This is due to intrinsic differences between the stopping powers of the two models.

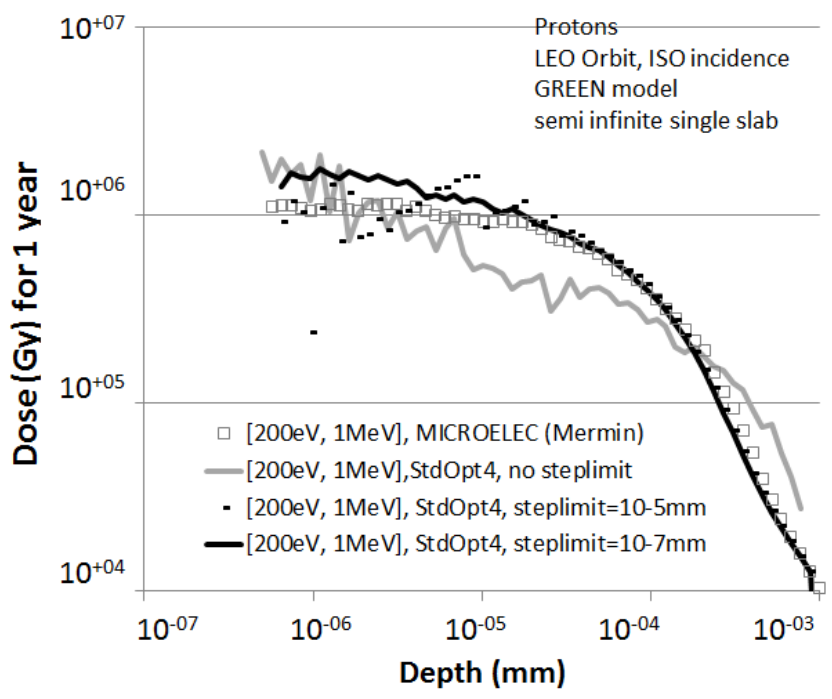

Fig. 8. Dose depth curve for LEO $\left(800 \mathrm{~km}, 9^{\circ}\right)$ protons. The G4EmStandardPhysics_option4 model with different step conditions are compared to MICROELEC discrete process.
This good behaviour of continuous processes is sustained by the fact that the dose deposited by low energy protons arriving at isotropic incidence is essentially driven by the penetration depth of the protons. The incoming particles, impinging the volume with an isotropic incidence, will reach lower depth for increasing angles of incidence. The average deposited dose is therefore smoothed over the volume bounded by the surface of the material and the path of the incident particles. For an isotropic incident environment, the shape of the dose depth curve is driven by the shape of the incident energetic spectrum. The dose, can be approximated without making a huge error, assuming very roughly, that the energy is deposited at a depth equal to the path of the protons. Similar conclusions can be drawn for electrons.

As a conclusion it is possible to calculate the dose depth profile for low energy incident particles at depths close to the surface with continuous processes. Care must just be taken to the step length chosen by the algorithm. Limiting the length of the step below $1 \mu \mathrm{m}$ depth is necessary to avoid big errors. But aside from this reservation, the continuous processes can be used to calculate the dose at the scale of few nanometers for both protons and electrons.

The following section presents a comparison of the dose depth profiles calculated for LEO (Spot) and GEO orbits using GREEN and AE8/AP8 environment models.

\section{LEO AND GEO MISSIONS DOSE DEPTH PROFILES}

The dose depth profiles have been calculated for LEO and GEO missions in a semi-infinite single slab, irradiated with an isotropic (Lambertian) incident flux. In order to limit the CPU time consumption, the calculations have been performed with G4EmStandardPhysics_option4 model and a step limit of 1 Angström. Some comparisons are shown at high energy with OMERE [7] and MULASSIS [14] toolkits.

In order to analyse the contribution to the dose of the low energy part of the spectrum, different cases have been compared to the AE8 and AP8 simulations. First, cut-off energies of $40 \mathrm{keV}$ and $100 \mathrm{keV}$, corresponding to the cut-off energies of AE8 and AP8, have been applied respectively for electrons and protons. As can be seen in the following figures for LEO spot orbit (Figs. 9 and 10), the dose depth profiles calculated are close to the AE8 and AP8 simulations, aside from the slight discrepancy between GREEN and AE8/AP8 models. The transport has been performed close to the surface at a depth of $1 \mathrm{~nm}$. It is far lower than for OMERE, which provides the dose beyond $10 \mu \mathrm{m}$. MULASSIS pushes the calculation down to $0.5 \mu \mathrm{m}$. One can notice on these figures the good agreement between our AE8/AP8 calculations and those performed with OMERE and MULASSIS.

Decreasing the low energy cut-off significantly changes the deposited dose close to the surface below $1 \mu \mathrm{m}$ depth. In the case of electrons (Fig. 9), the low energy part of the GREEN spectrum between $1 \mathrm{keV}$ and $40 \mathrm{keV}$ multiplies the dose at the surface by a factor of $\sim 100$. On the other hand, the portion of the spectrum below $1 \mathrm{keV}$ [200eV-1 keV] does not add any significant contribution to the dose at the surface. This is entirely consistent with the electron fluxes presented in Fig. 1 which are quite low below $1 \mathrm{keV}$. 


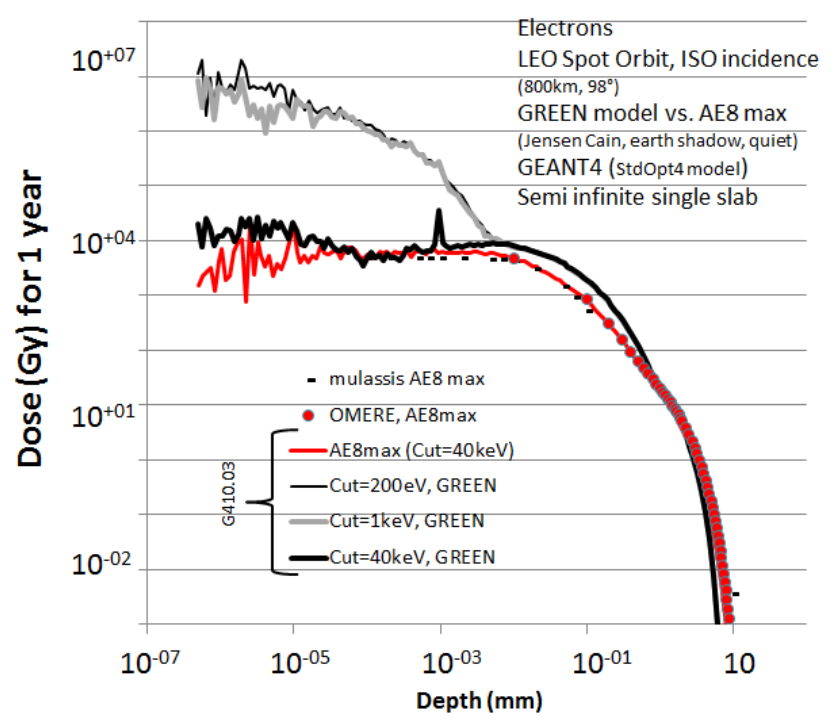

Fig. 9. Dose depth profile for incident electrons at LEO Spot orbit. Comparison GREEN vs. AE8. The incident spectrum has been cut at three different low energies $(200 \mathrm{eV}, 1 \mathrm{keV}$, and $40 \mathrm{keV})$.

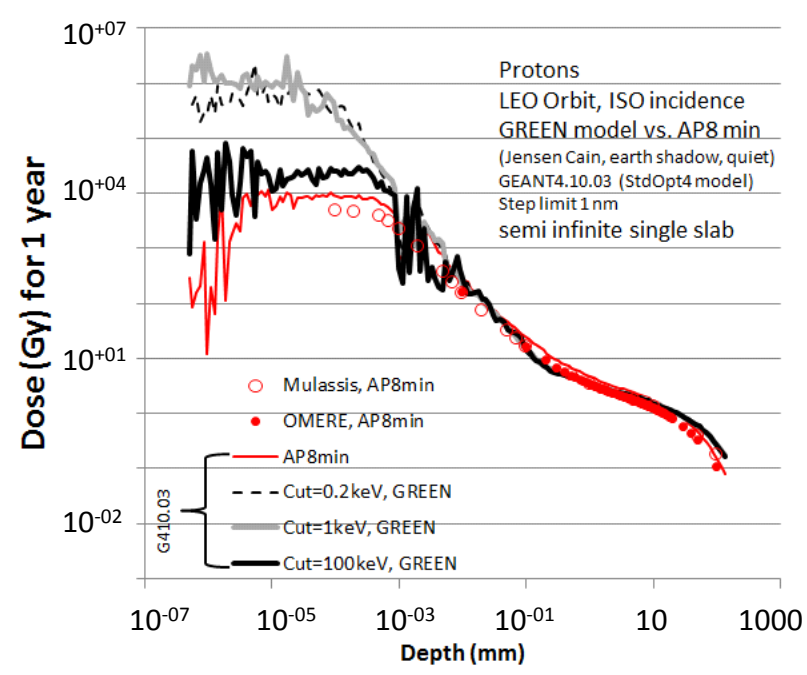

Fig. 10 . Dose depth profile for incident protons at LEO Spot orbit. Comparison GREEN vs. AE8. The incident spectrum has been cut at three different low energies $(200 \mathrm{eV}, 1 \mathrm{keV}$, and $100 \mathrm{keV})$.

For protons, the comments are quite similar (Fig. 10). The energy distributions of GREEN and AP8 models are very close above $100 \mathrm{keV}$, which leads to very close dose profiles beyond $1 \mu \mathrm{m}$. Adding the contribution of the spectrum below $100 \mathrm{keV}$, enhances the dose deposited at the surface $(1 \mathrm{~nm})$ by a factor of 100 for the LEO orbit.

Similar conclusions can be drawn for GEO orbits (Figs. 11 and 12). For protons, adding the contribution of the spectrum below $100 \mathrm{keV}$ enhances the surface dose by a big decade for the GEO orbit (Fig. 12). A factor of nearly 100 is observed for electrons (Fig. 11). Knowing that some changes in material properties can be observed from $\sim 10^{+05} \mathrm{~Gy}$, the impact on the assessment of the radiation resistance of space material could be important.

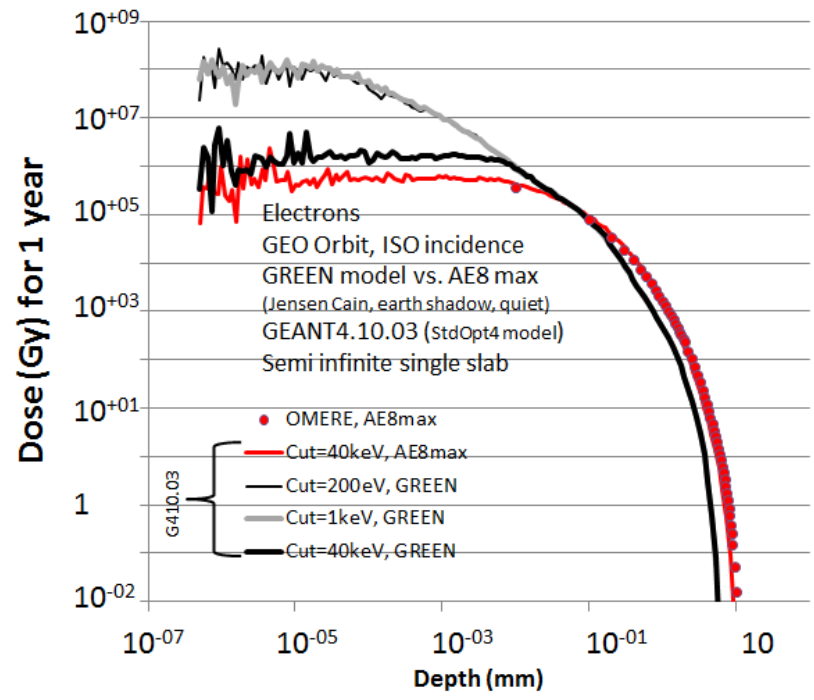

Fig. 11. Dose depth profile for incident protons at LEO Spot orbit. Comparison GREEN vs. AE8. The incident spectrum has been cut at three different low energies $(200 \mathrm{eV}, 1 \mathrm{keV}$, and $100 \mathrm{keV})$.

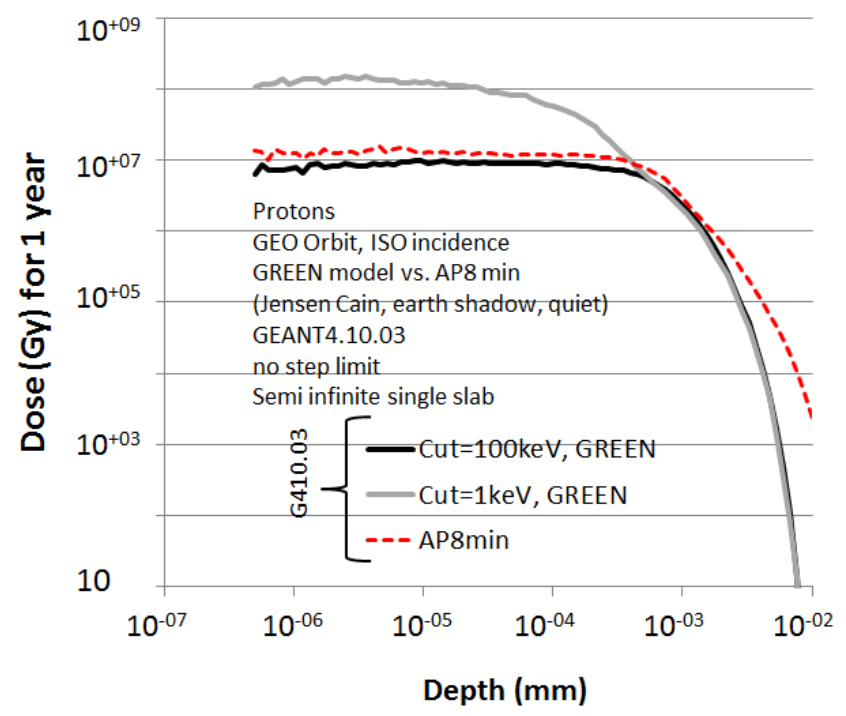

Fig. 12. Dose depth profile for incident protons at GEO orbit (GREEN vs. AE8). Different cutoff energies are applied (1 keV and $100 \mathrm{keV})$.

\section{CONCLUSION}

Satellites' external coatings, such as white paints, Kapton, OSR, MLI are exposed to high radiation levels, which are poorly estimated in the very near surface $(<1 \mu \mathrm{m})$, where their thermo-optical properties lay. Below $1 \mu \mathrm{m}$ depth, the radiation level cannot be estimated precisely, first because, low energy particle fluxes are not provided by current environment models such as AE8 and AP8. These models have cutoff energies of respectively $40 \mathrm{keV}$ and $100 \mathrm{keV}$. Second, below some $\mathrm{keV}$, the continuous processes used in conventional transport codes present some limitations. New environment models (GREEN, AE9/AP9), combined with discrete transport code (GEANT4/Microelec) allows now to address the question of the surface dose level. In that work, the influence of low energy contribution on the dose deposited at the surface of materials subject to space environments has been investigated. 
First, it is shown that discrete transport processes are more suited than continuous ones for the transport of low energy particles (e- $<\sim \mathrm{keV}, \mathrm{p}+<\sim 10 \mathrm{keV}$ ). But, due to an averaging mechanism a reasonable accuracy is found within the surface dose using continuous processes. Care must be taken to the step length when using the latter models.

Second, dose depth profiles calculated with AE8 and AP8 models have been compared to GREEN model calculations. Both GEO and LEO SPOT like orbits have been studied. Roughly, electrons having energies below the AE8 cut-off (40 $\mathrm{keV}$ ) increase the surface dose by a factor of $\sim 100$, while protons below the $100 \mathrm{keV}$ AP8 cut-off increase the dose by a factor of $\sim 10$. According to GREEN model the contribution to the surface dose of electrons becomes comparable to the one of protons, while it is fairly dominated by protons when using AE8/AP8

All this work has been performed for silicon material. But at low energy different materials can behave quite differently according to their nature (metals, insulators...). In addition the surface state with its roughness, the presence of dangling bonds, or the band structure distortion, are not taken into account. In conclusion, the aluminium dose equivalence commonly used in the space domain should be studied in more detail in the scope of surface material degradation.

\section{REFERENCES}

[1] SRIM- The stopping and range of ions in matter [Online]. Available: http://www.srim.org/, Accessed January 27, 2020.

[2] J. F. Ziegler, M. D. Ziegler and J. P. Biersack, "SRIM - The stopping and range of ions in matter (2010)," Nucl. Instrum. Methods Phys. Rev. B, vol. B268, no. 11-12, pp.1818-1823, Jun. 2010.

[3] ESTAR- Stopping and range tables for electrons [Onl ne]. Available: https://physics.nist.gov/PhysRefData/Star/Text/ESTAR.html, Accessed January 27, 2020.

[4] PSTAR- Stopping and range tables for protons https://physics.nist.gov/PhysRefData/Star/Text/PSTAR.html, Accessed January $27,2020$.

[5] S. M. Seltzer, SHIELDOSE, "A Computer Code for Space-Shielding Radiation Dose Calculations," National Bureau of Standards, NBS Technical Note 1116, U.S. Government Printing Office, Washington, D.C., May 1980.

[6] S. M. Seltzer, "Updated calculations for routine space-shielding radiation dose estimates: SHIELDOSE-2," NIST Publication NISTIR 5477, Gaithersburg, MD., Dec. 1994.

[7] OMERE: Outil de Modélisation de l'Environnement Radiatif Externe, version 4.2. [Online]. Available:http://www.trad.fr/OMERE-14.html Accessed July 27, 2017

[8] T. Bortfeld and W. Schlegel, "An analytical approximation of depthdose distributions for therapeutic proton beams," Phys Med Biol. Vol 41, no. 8, pp. 1331-1339, Apr. 1996.

[9] A.J. Tylka, J. H. Adams, Jr., P. R. Boberg, B. Brownstein, W. F. Dietrich, E. O. Flueckiger, E. L. Petersen, M. A. Shea, D. F. Smart, and E. C. Smith, "CREME96: A Revision of the Cosmic Ray Effects on Micro-Electronics Code," IEEE Trans. Nucl. Sci., vol. 44, no. 6, pp. 2150-2160, Dec. 1997.

[10] CREME [Online]. Available: https://creme.isde.vanderbilt.edu/, Accessed January 27, 2020.

[11] T. Jordan, "NOVIVE a radiation transport/shielding code," E.M.P. Consultants report, Jan. 1990.

[12] EMPC- Novice software, [Online]. Available: https://empc.com/novice-software/ Accessed January 27, 2020.

[13] FASTRAD - 3D radiation software [Online]. Available: https://www.fastrad.net/ Accessed January 27, 2020.

[14] MULASSIS - Multi-layered shielding simulation software [Online]. Available: https://www.spenvis.oma.be/help/models/mulassis.html Accessed January 27, 2020.

[15] SPENVIS - The space environment information system [Online]. Available : https://www.spenvis.oma.be/ Accessed January 27, 2020.
[16] S. Agostinelli et al., "Geant 4 a simulation toolkit," Nucl. Instrum. Methods Phys. Rev. A, vol. A506, no. 3, pp.250-303, Feb. 2003.

[17] GEANT4 - A simulation toolkit [Online]. Available https://geant4.web.cern.ch/ Accessed January 27, 2020.

[18] A Roggero, E Dantras, T Paulmier, C Tonon, S Lewandowski, S Dagras and D Payan, "Electrical conductivity of a silicone network upon electron irradiation: influence of formulation," J. Phys. D: Appl. Phys. Vol. 49, no. 50, art. no. 505303, Nov. 2016.

[19] S. Lewandowski, S. Duzelier, J. Eck, S. Dagras, C. Tonon, H. Jochem, P. Jouanne, E. Laurent and J.M. Desmarres, "Particle Flux Effects on Physicochemical Polymer Degradations," J. Spacecr. Rockets. Vol. 53, no. 6, pp. 1146-1151, Aug. 2016.

[20] J. Vette, "Models of the trapped radiation environment, Volume I: Inner zone protons and electron," NASA SP-3024, Washington, D.C., Jan. 1966.

[21] AE8/AP8 - Radiation belt models [Online]. Available : https://ccmc.gsfc.nasa.gov/modelweb/models/trap.php Accessed January $27,2020$.

[22] G. P. Ginet, T.P. O’Brien, S.L. Huston, W.R. Johnston, T.B. Guild, R. Friedel, C.D. Lindstrom,C.J. Roth, P. Whelan, R.A. Quinn, D. Madden, S. Morley and Yi-Jiun Su, "AE9, AP9 and SPM:New Models for Specifying the Trapped Energetic Particle and Space Plasma Environment," Space Science Reviews, vol. 179, no. 1-4, pp. 579-615, Mar. 2013.[http://dx.doi.org/10.1007/s11214-013-9964-y]

[23] AE9/AP9/SPM - Radiation belt and space plasma specification models[Online].Available:https://www.vdl.afrl.af.mil/programs/ae9ap9, Accessed January 27, 2020.

[24] A. Sicard, D. Boscher, S. Bourdarie, D. Lazaro, D. Standarovski and R. Ecoffet, "GREEN: the new Global Radiation Earth Environment model," Ann. Geophys., vol. 36, pp. 953-967, 2018. https://doi.org/10.5194/angeo-36-953-2018, Jul. 2018

[25] J.Baró, J.Sempau, J.M.Fernández-Varea and F.Salvat "PENELOPE: An algorithm for Monte Carlo simulation of the penetration and energy loss of electrons and positrons in matter," Nucl. Instr. And Meth., vol. 100, no.1, pp. 34-46, May 1995.

[26] M. Raine, A. Valentin, M. Gaillardin and P. Paillet, "Improved simulation of ion track structures using new Geant4 models - Impact on the modeling of advanced technologies response," IEEE Trans. Nucl. Sci., vol. 59, no. 6, pp. 2697 - 2703, Oct. 2012.

[27] M. Raine, M. Gaillardin and P. Paillet, "Geant4 physics processes for silicon microdosimetry simulation: Improvements and extension of the energy-range validity up to $10 \mathrm{GeV} /$ nucleon," Nucl. Instr. and Meth. in Phys. Res. B, vol. 325, no.15, pp. 97-100, Apr. 2014.

[28] A. Valentin, M. Raine, J.-E. Sauvestre, M. Gaillardin and P. Paillet, "Geant4 physics processes for microdosimetry simulation: very low energy electromagnetic models for electrons in silicon," Nucl. Instr. and Meth. in Phys. Res. B, vol. 288, no. 1, pp. 66-73, Oct. 2012.

[29] A. Valentin, M. Raine, M. Gaillardin and P. Paillet, "Geant4 physics processes for microdosimetry simulation: very low energy electromagnetic models for protons and heavy ions in silicon," Nucl. Instr. and Meth. in Phys. Res. B, vol. 287, no. 15, pp. 124-129, Sept. 2012

[30] J. Pierron, C. Inguimbert, M. Belhaj, M. Raine and J. Puech "Ionizing Doses Calculations for Low Energy Electrons in Silicon and Aluminum," IEEE Trans. Nucl. Sci., vol. 64, no. 8, pp. 2340-2348, Feb. 2017.

[31] J. Pierron, C. Inguimbert, M. Belhaj, T. Gineste, J. Puech and M. Raine, "Electron emission yield for low energy electrons: Monte Carlo simulation and experimental comparison for $\mathrm{Al}, \mathrm{Ag}$ and $\mathrm{Si}$," J. of Appl. Phys., vol. 121 art. no. 215107, May 2017.

[32] P. Caron, C. Inguimbert; L. Artola; N. Chatry, N. Sukhaseum, R. Ecoffet and F. Bezzera, "Physical Mechanisms Inducing Electron Single-Event Upset," IEEE Trans. Nucl. Sci., vol. 65, no. 8, pp. 17591767, Mar. 2018.

[33] P. Caron, C. Inguimbert, L. Artola, F. Bezerra and R. Ecoffet, "New SEU modeling method for calibrating target system to multiple radiation particles," IEEE Trans. Nucl. Sci., vol. 65, no. 8, pp. 17591767, Nov. 2019.

[34] E.J. Kobetich, and R. Katz, "Electron energy dissipation," Nucl. Instr. And Meth., vol. 71, no.2, pp. 226-230, Feb.1969.

[35] F.Salvat, A. Jablonski, and C. J. Powel, "Elsepa-Dirac partial-wave calculation of elastic scattering of electrons and positrons by atoms, positive ions and molecules," Comp. Phys. Comm., vol. 165, no. 2, pp.157-190, Jan. 2005. 